13.4

\title{
Многочастотное излучение киловаттного уровня мощности в непрерывной винтовой гирорезонансной лампе обратной волны К-диапазона с внешними отражениями
}

\author{
(C) Р.М. Розенталь, С.В. Самсонов, А.А. Богдашов, И.Г. Гачев, М.Ю. Глявин
}

Институт прикладной физики РАН, Нижний Новгород, Россия

E-mail: rrz@ipfran.ru

Поступило в Редакцию 14 августа 2020г.

В окончательной редакции 30 ноября 2020г.

Принято к публикации 30 ноября 2020 г.

\begin{abstract}
За счет введения внешних отражений в гирорезонансной лампе обратной волны на основе винтового гофрированного волновода экспериментально реализованы режимы одновременного возбуждения нескольких продольных мод совокупной электродинамической системы. Получено излучение диапазона $24 \mathrm{GHz}$ co средней мощностью $0.9-1.2 \mathrm{~kW}$ с характерным расстоянием между спектральными компонентами 96-120, 232-280 и 464-640 MHz, соответствующими возбуждению продольных мод с различными индексами.
\end{abstract}

Ключевые слова: гиро-ЛОВ, автомодуляционные режимы, многочастотный нагрев плазмы.

DOI: 10.21883/PJTF.2021.06.50750.18510

В настоящее время активно исследуются процессы двухчастотного СВЧ-нагрева плазмы, которые требуют непрерывного излучения с уровнем мощности как минимум в сотни ватт [1-4]. В сантиметровом диапазоне для этого используются приборы с прямолинейными электронными потоками, такие как клистроны и лампы бегущей волны (ЛОВ). Однако необходимость транспортировки электронных пучков на расстояния порядка длины волны от границ электродинамической системы в значительной степени сдерживает продвижение приборов данного класса в область более высоких частот, где подобные исследования могли бы быть проведены с плазмой большей плотности. В связи с этим большой интерес представляют приборы гирорезонансного типа, где винтовой электронный поток может взаимодействовать с модами, поля которых сосредоточены вдали от поверхности электродинамической системы [5]. В настоящее время на основе источников такого типа реализованы технологические комплексы, рассчитанные на непрерывную работу и обеспечивающие выходную мощность $20 \mathrm{~kW}$ на частоте $45 \mathrm{GHz}$ и более $2.5 \mathrm{~kW}$ на частоте $300 \mathrm{GHz}$ [6]. Это как минимум на порядок превышает значения, достигнутые в классических СВЧ-приборах с прямолинейными электронными потоками.

Вместе с тем ввиду довольно значительной энергетики электронных потоков и необходимости создания сильных магнитных полей гирорезонансные приборы являются достаточно громоздкими и дорогостоящими устройствами, что ограничивает возможности одновременного использования нескольких таких источников. Одним из решений является применение многочастотных автомодуляционных режимов генерации, возникающих при значительных уровнях надкритичности (превышения рабочего тока над стартовыми значениями) [7-10]. При этом введение отражений выходного излучения позволяет значительно снизить бифуркационные значения токов, что делает возможным получение многочастотных режимов генерации без существенных изменений энергетических параметров электронного потока [11-14]. В случае сильных отражений спектральные линии излучения в режиме периодической автомодуляции близки к резонансным частотам совокупной электродинамической системы, образованной электродинамической системой и внешним отражателем. Это достаточно универсальное свойство СВЧ-генераторов характерно, в частности, и для генераторов на основе ЛОВ с внешним отражателем [15-17].

В связи с этим представляет интерес исследование возможностей получения многочастотного излучения, пригодного для задач нагрева плазмы, на основе гирорезонансных ЛОВ (гиро-ЛОВ) с винтовым гофрированным волноводом. Такие генераторы привлекательны в первую очередь в связи с возможностью плавной и широкополосной перестройки частоты излучения в пределах одной поперечной моды электродинамической системы при изменении циклотронной частоты. При этом по сравнению с генераторами на основе гладких волноводов при тех же параметрах электронного пучка винтовой волновод позволяет в 2-3 раза расширить полосу перестройки частоты.

Цель настоящей работы состоит в экспериментальной реализации многочастотных режимов генерации в ЛОВ такого типа и определении основных характеристик полученного излучения. Исследования проводились с использованием непрерывной винтовой гиро-ЛОВ с центральной рабочей частотой около $24 \mathrm{GHz}$ [18]. Схема основных компонентов системы представлена на рис. 1. Электронная пушка магнетронно-инжекторного типа $(1,2$ на рис. 1$)$ формировала винтовой электронный пучок с радиусом ведущих центров $1.7 \mathrm{~mm}$, энергией 


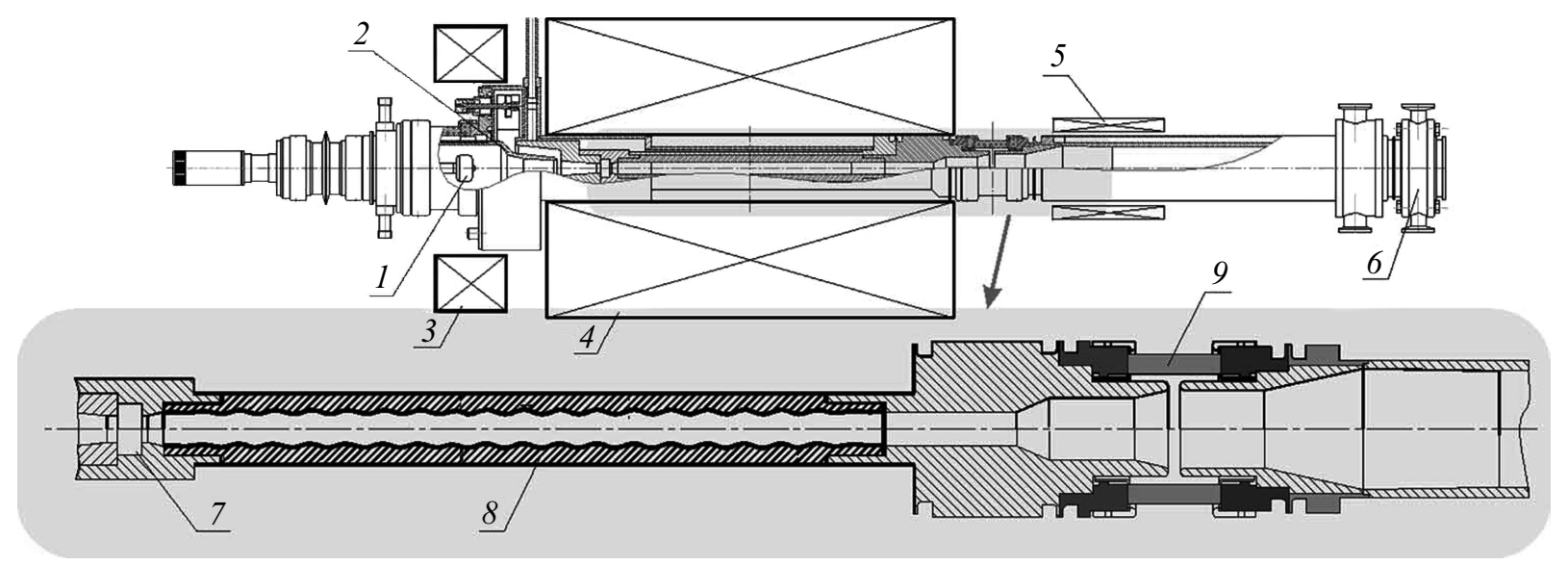

Рис. 1. Схема экспериментального стенда. 1 - катод, 2 - анод, 3 - катодная катушка, 4 - соленоид ведущего магнитного поля, 5 - коллекторная катушка, 6 -- выходное рассогласованное окно с системой охлаждения, 7 - резонансный отражатель, 8 - винтовой гофрированный волновод, 9 - коллекторный изолятор.

до $20 \mathrm{keV}$, током до $2 \mathrm{~A}$ и расчетным питч-фактором (отношением поперечной осцилляторной скорости к продольной) 1.2-1.5 при разбросе поперечных скоростей 10-15\%. Ведущее магнитное поле величиной до $0.5 \mathrm{~T}$ формировалось „теплым“ соленоидом 4 на постоянном токе с жидкостным охлаждением. Рабочее магнитное поле менялось в пределах 0.4-0.5 Т, при этом сохранение заданной величины питч-фактора частиц обеспечивалось соответствующей подстройкой тока дополнительной катодной катушки 3. Для осаждения пучка на коллектор использовалась коллекторная катушка 5 , создающая встречное по отношению к основному магнитное поле.

Волновод 8 с трехзаходной винтовой гофрировкой со средним радиусом $5.65 \mathrm{~mm}$, периодом гофрировки $11.7 \mathrm{~mm}$ и длиной $180 \mathrm{~mm}$ обеспечивал связь бегущей моды $\mathrm{TE}_{1,1}$ и квазикритической моды $\mathrm{TE}_{2,1}$ обратного вращения и их взаимодействие с электронным пучком на второй гармонике циклотронной частоты. При этом групповая скорость рабочей волны составляла $(0.2-0.25) c$. Для отражения возбуждаемой волны от катодного конца пространства взаимодействия использовался резонансный отражатель 7, выполненный в виде канавки прямоугольного профиля, представляющей собой закрытый резонатор для моды $\mathrm{TM}_{1,1}$. За счет дифракционной связи с запертой модой $\mathrm{TM}_{1,1}$ падающая на такую канавку рабочая волна $\mathrm{TE}_{1,1}$ эффективно отражается на частотах, близких к соответствующей резонансной частоте. При этом в том случае, когда критическая частота, соответствующая минимальному радиусу канавки, ниже частоты падающей волны на $30-40 \%$, полоса отражения может быть довольно значительной. Так, указанный отражатель обеспечивал коэффициент отражения по мощности более 95\% в полосе частот от 22 до $26 \mathrm{GHz}$.

Коллекторный изолятор 9 был рассчитан на работу при статическом напряжении до $10 \mathrm{kV}$ и обеспечивал возможность реализации режимов с рекуперацией
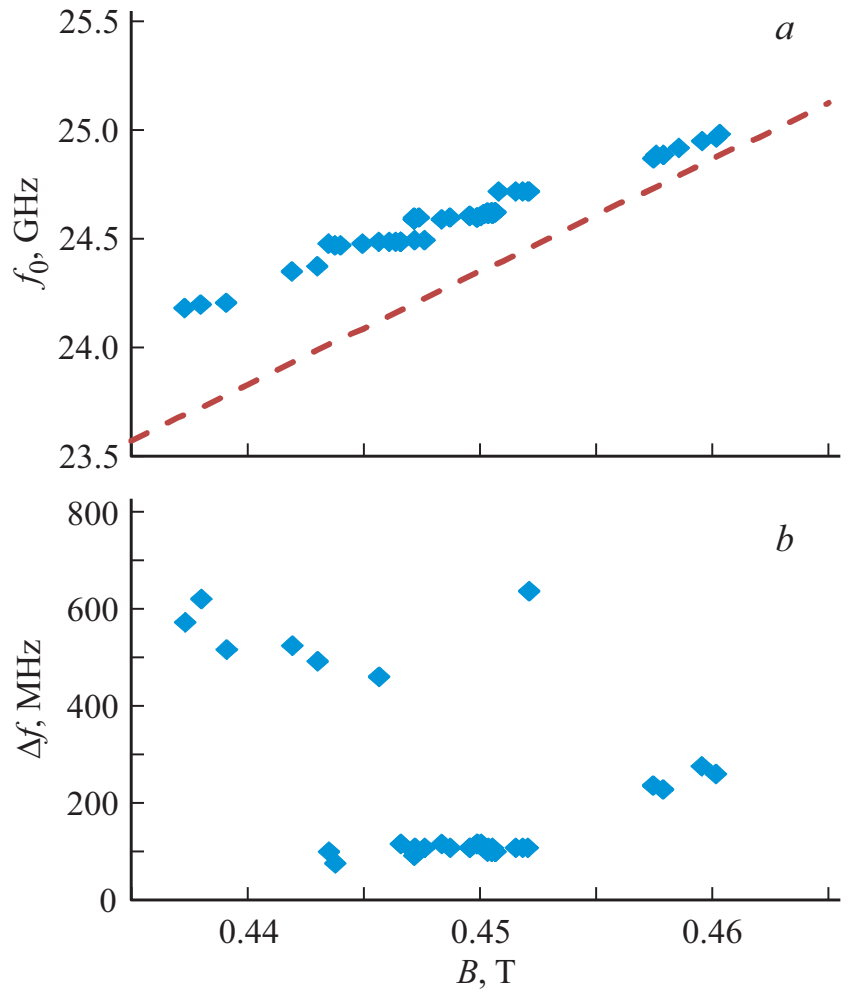

Рис. 2. Экспериментальные зависимости от магнитного поля: $a$ - центральной частоты генерации $f_{0}$ (штриховая линия - расчетная частота точного синхронизма гиро-ЛОВ); $b-$ расстояния между соседними линиями спектра $\Delta f$.

энергии электронного потока (в данных экспериментах указанная возможность не использовалась). Для снижения потерь мощности выходного излучения использовалась система цилиндрических и конических участков, на которых рабочая волна $\mathrm{TE}_{1,1}$ преобразовывалась в набор мод, суммарное поле которых в области волноводного разрыва было сосредоточено далеко от стенок. 

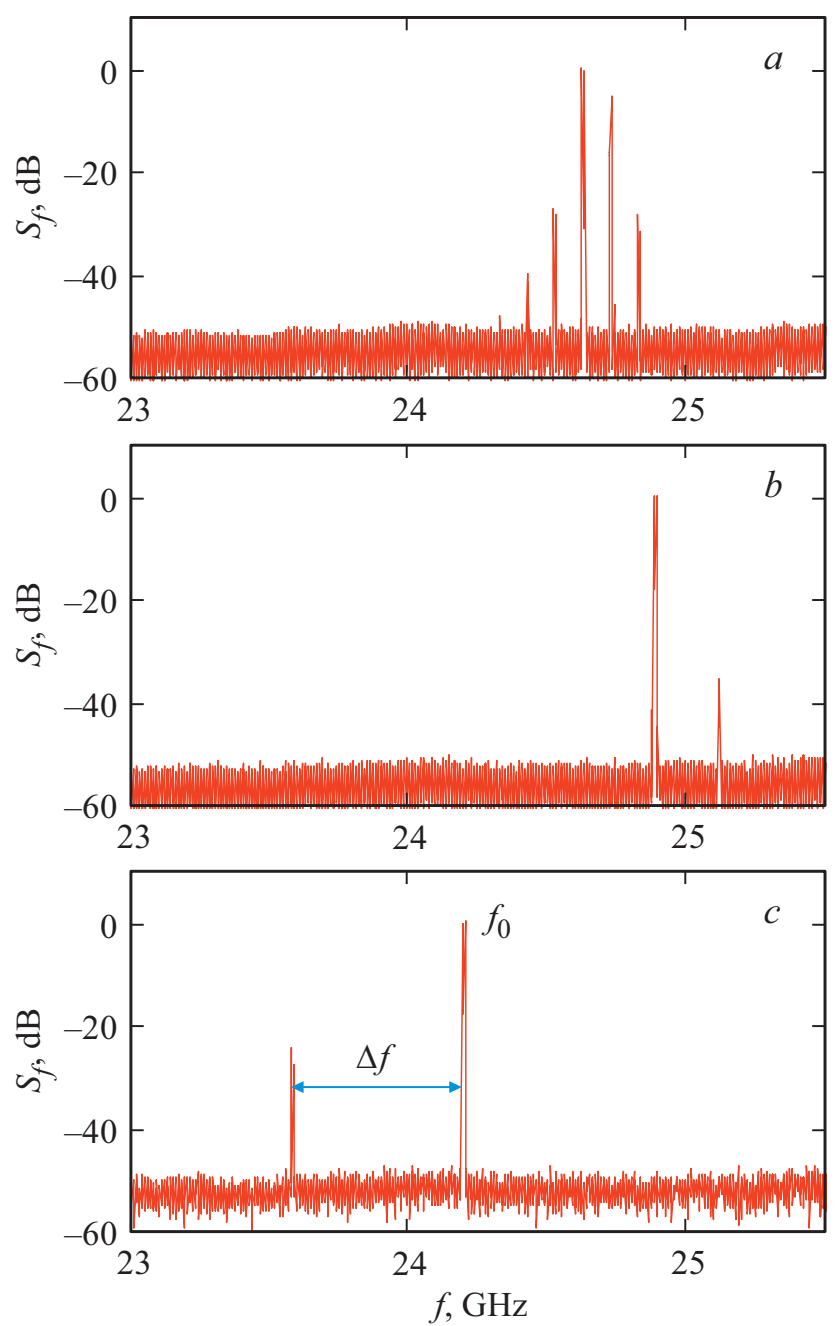

Рис. 3. $a-$ спектр излучения при возбуждении соседних мод с числом продольных вариаций $n$ и $n+1(B=0.4506 \mathrm{~T}) ; b-$ мод с числом продольных вариаций $n$ и $n+2(B=0.4578 \mathrm{~T})$; $c-n$ и $n+6(B=0.438 \mathrm{~T})$.

В свою очередь на выходе данного участка этот набор мод преобразовывался обратно в поле волны $\mathrm{TE}_{1,1}$. В соответствии с расчетами коэффициент передачи данной системы в диапазоне $23-25 \mathrm{GHz}$ составлял не менее 95\%. Выходное окно 6, аналогичное типичным окнам технологических гиротронов, было изготовлено из нитрида бора (BN) [19]. Рассогласование окна было реализовано за счет уменьшения его толщины, так что в рабочем диапазоне частот коэффициент отражений составил порядка $30 \%$ от мощности падающего излучения.

Регистрация спектра излучения гиро-ЛОВ проводилась с помощью спектроанализатора Keysight N9010A с верхней границей диапазона частот $44 \mathrm{GHz}$. Выходная мощность определялась при помощи калориметрического измерителя, являвшегося одновременно согласованной нагрузкой и обеспечивавшего относительную точность измерения около $10 \%$ при уровне мощности выше $1 \mathrm{~kW}$. Центральная частота генерации была близка к расчетной частоте точного синхронизма (рис. 2,a). Режимы периодической автомодуляции наблюдались в диапазоне изменения ведущего магнитного поля от 0.437 до $0.460 \mathrm{~T}$ (рис. 2,b). При этом в середине полосы по магнитному полю частота автомодуляции была сосредоточена в интервале значений $\Delta f=96-120 \mathrm{MHz}$, близких к обратному времени прохода излучения по цепи обратной связи $1 / T \approx 110 \mathrm{MHz}$. При увеличении магнитного поля наблюдались режимы с $\Delta f=232-280 \mathrm{MHz}$, а при уменьшении - с $\Delta f=464-640 \mathrm{MHz}$. Примеры спектров выходного излучения с частотами автомодуляции 104, 232 и $624 \mathrm{MHz}$ и средней мощностью 1.2, 1.6 и $0.9 \mathrm{~kW}$ соответственно представлены на рис. 3 .

Наблюдаемая динамика системы соответствует так называемому частотному механизму возникновения автомодуляции, связанному с одновременным возбуждением нескольких продольных мод резонатора, образованного отражателем 7 и рассогласованным окном 6. При этом случай $\Delta f=96-120 \mathrm{MHz}$ соответствует возбуждению соседних мод с числом продольных вариаций $n$ и $n+1$, $\Delta f=232-280 \mathrm{MHz}$ - мод с числом продольных вариаций $n$ и $n+2, n+3, \Delta f=464-640 \mathrm{MHz}-n$ и $n+5$, $n+6$.

С практической точки зрения наиболее интересным представляется режим возбуждения соседних продольных мод с соразмерной интенсивностью спектральных компонент и средней мощностью, превышающей $1 \mathrm{~kW}$ (рис. 3,a). Такое излучение может найти применение в системах многочастотного нагрева плазмы с близкими значениями частот [2].

\section{Финансирование работы}

Работа выполнена при поддержке Российского фонда фундаментальных исследований (грант № 19-08-00955).

\section{Конфликт интересов}

Авторы заявляют, что у них нет конфликта интересов.

\section{Список литературы}

[1] V. Skalyga, I. Izotov, T. Kalvas, H. Koivisto, J. Komppula, R. Kronholm, J. Laulainen, D. Mansfeld, O. Tarvainen, Phys. Plasmas, 22 (8), 083509 (2015). DOI: 10.1063/1.4928428

[2] A. Kitagawa, S. Biri, Y. Kato, M. Muramatsu, R. Rácz, W. Takasugi, in 22nd Int. Workshop on ECR ion sources (ECRIS) (Busan, Korea, 2016), p. 55-58. DOI: 10.18429/JACoW-ECRIS2016-WEAO03

[3] E. Naselli, D. Mascali, M. Mazzaglia, S. Biri, R. Rácz, J. Pálinkás, Z. Perduk, A. Galatá, G. Castro, L. Celona, S. Gammino, G. Torrisi, Plasma Sources Sci. Technol., 28 (8), 085021 (2019). DOI: 10.1088/1361-6595/ab32f9

[4] A.G. Shalashov, E.D. Gospodchikov, I.V. Izotov, Plasma Phys. Control. Fusion, 62 (6), 065005 (2020). DOI: $10.1088 / 1361-6587 / \mathrm{ab} 7 \mathrm{f9} 9$

[5] M.I. Petelin, J. Infrared Milli. Terahz. Waves, 38 (11), 1387 (2017). DOI: 10.1007/s10762-017-0423-9 
[6] M. Thumm, J. Infrared Milli. Terahz. Waves, 41 (1), 1 (2020). DOI: https://doi.org/10.1007/s10762-019-00631-y

[7] N.S. Ginzburg, G.S. Nusinovich, N.A. Zavolsky, Int. J. Electron., 61 (6), 881 (1986). DOI: $10.1080 / 00207218608920927$

[8] T.H. Chang, S.H. Chen, L.R. Barnett, K.R. Chu, Phys. Rev. Lett., 87 (6), 064802 (2001). DOI: 10.1103/PhysRevLett.87.064802

[9] S. Alberti, J.-Ph. Ansermet, K.A. Avramides, F. Braunmueller, P. Cuanillon, J. Dubray, D. Fasel, J.-Ph. Hogge, A. Macor, E. de Rijk, M. da Silva, M.Q. Tran, T.M. Tran, Q. Vuillemin, Phys. Plasmas, 19 (12), 123102 (2012).

DOI: $10.1063 / 1.4769033$

[10] Р.М. Розенталь, А.Э. Федотов, Н.С. Гинзбург, И.В. Зотова, А.Б. Волков, С.В. Самсонов, Е.С. Семенов, А.С. Сергеев, Письма в ЖТФ, 45 (10), 38 (2019). DOI: $10.21883 /$ PJTF.2019.10.47755.17746

[11] Н.С. Гинзбург, Н.И. Зайцев, Е.В. Иляков, И.С. Кулагин, Р.М. Розенталь, Письма в ЖТФ, 28 (9), 85 (2002).

[12] R.M. Rozental, N.S. Ginzburg, M.Y. Glyavin, A.S. Sergeev, IEEE Trans. Microwave Theory Tech., 54 (6), 2741 (2006). DOI: $10.1109 /$ TMTT.2006.874876

[13] М.В. Белоглазкина, А.А. Короновский, А.Е. Храмов, Письма в ЖТФ, 32 (12), 1 (2006).

[14] Р.М. Розенталь, Н.С. Гинзбург, Н.И. Зайцев, Е.В. Иляков, И.С. Кулагин, ЖТФ, 76 (1), 82 (2006).

[15] Н.М. Рыскин, В.Н. Титов, Изв. вузов. Радиофизика, 44 (10), 860 (2001).

[16] Р.М. Розенталь, Н.С. Гинзбург, А.С. Сергеев, Изв. вузов. Радиофизика, 50 (12), 1043 (2007).

[17] N.M. Ryskin, V.N. Titov, O.V. Umantsiva, IEEE Trans. Plasma Sci., 44 (8), 1270 (2016). DOI: 10.1109/TPS.2016.2517002

[18] S.V. Samsonov, G.G. Denisov, V.L. Bratman, A.A. Bogdashov, M.Yu. Glyavin, A.G. Luchinin, V.K. Lygin, M.K. Thumm, IEEE Trans. Plasma Sci., 32 (3), 884 (2004). DOI: $10.1109 /$ TPS.2004.828871

[19] V.V. Parshin, Int. J. Infrared Milli. Waves, 15 (2), 339 (1994). DOI: 10.1007/BF02096245 